\title{
Predicting Collisionless Equlibria in Dark Matter Simulations
}

\begin{abstract}
Robert J. Ragan
Department of Physics, University of Wisconsin - La Crosse ${ }^{1}$

By using a transformation from phase-space variables to a set of orthogonal Hermite-Legendre polynomials, the dynamics of onedimensional self-gravitating systems has been reduced to a linear set of equations. This transformation changes the long-range forces into local interactions in the new problem. This makes it possible to determine the steady-state solutions by projecting the initial conditions into the timeindependent solutions.
\end{abstract}

\section{Introduction}

Astrophysicists have made significant progress in forming a consistent picture of how large-scale structures (namely galaxies and galaxy clusters) form through the use of $N$-body computer simulations. In fact simulations are the main tool for determining the role dark matter halos play in structure and evolution of galaxies. These models vary in their level of detail - some include only gravitational interactions, while others include additional gas dynamics and stellar evolution. Although these simulations reproduce observed self-gravitating objects, their behavior is not fully characterized and the driving mechanisms in their evolution are not fully understood. Some questions that still need to be answered are: What determines the equilibrium state of such a system? How does the final state depend on the initial conditions? What features are universal? What role does entropy play in the relaxation process?

Although realistic models do enable us to reproduce observed structures, their complexity makes analysis very difficult and mostly empirical in nature. For this reason, my UW-L colleague Eric Barnes, and I have been studying one-dimensional collisionless systems. These systems relax to quasi-stationary equilibria via self-gravity in a very similar fashion to 3-d systems, but are much easier to analyze. In particular, their phase-space distributions can be linearized and expanded in terms of orthogonal polynomials. The equations of motion of the expansion coefficients can be written as a sparse linear system. Diagonalization of the equations of motion yields the spectrum and modes of the dynamics as well as the time-independent solutions.

This approach allows us to predict the final equilibrium state from the initial conditions. But more than that, it gives a very illuminating picture of the phase-space motion of the system in terms of the evolution of the expansion coefficients whose equations of motion are local in that couplings are only between nearest-neighboring coefficients. This gives a compelling picture of conserved quantities like energy and fine-grained entropy that evolve via continuity-type equations in coefficient space. Furthermore, higher-order expansions should yield information

\footnotetext{
${ }^{1}$ This work was supported a WSGC Research Infrastructure Program grant.
} 
about correlations between different modes, such as how they develop and how they relate to the energy and entropy of the system. Taken together with $\mathrm{N}$-body simulations this investigation should yield a deeper understanding of the role of dark matter halos in galaxy formation.

\section{Background}

There is a broad consensus among astrophysicists and cosmologists that visible matter makes up only $5 \%$ of the mass-energy of the universe. Another $25 \%$ is thought to be weakly-interacting dark matter (Spergel et al., 2003), and the remaining 70\% is hypothesized to be dark energy. The picture has been inferred from the velocity curves of star orbits within galaxies (Rubin \& Ford,

1970), the motion of galaxies within clusters (Zwicky, 1937), gravitational lensing (Clowe et al., 2006), and rate of expansion of the universe.

In particular studies of individual galaxies indicate that the observed velocity curves are due to halos of dark matter that surrounds galaxies. $N$-body simulations of galaxies can involve billions of point masses interacting via gravity, sometimes with additional gas physics and stellar evolution. These studies have been successful in reproducing typically observed galactic structures (density profiles, velocity curves, etc.) from "cosmological" initial conditions, i.e. nearly uniform, expanding mass distributions (Navarro et al., 1997, 2004).

Dark matter interacts via gravity but its motion is assumed to be collisionless. A natural question that arises is: how does a dark matter halo relax? Computer simulations indicate that relaxation is generally incomplete, that different initial conditions lead to different equilibria. In fact, for 3$\mathrm{d}$ systems there is no self-gravitating thermal equilibrium with a uniform temperature (LyndenBell 1967), and in general systems partially relax to an equilibrium state belonging to continuum of quasi-stationary states (Hohl \& Felix, 1967).

Numerous attempts have been made to understand the details of collisionless relaxation processes (Taylor \& Navarro, 2001; Hansen \& Moore, 2006; Lithwick \& Dalal, 2011). Although they have added new insights, none of them have provided a comprehensive physical picture. Progress in this direction is the motivation of this project.

Instead of tackling the full 3-d problem head-on, we have chosen to study 1-d self-gravitating systems. These systems are a lot easier to analyze and simulate yet they possesses many of the essential features of interest, like long-range forces, self-gravitation, and collisionless relaxation. On the other hand the 1-d problem lacks 3-d properties like angular momentum and tidal forces.

The collisionless phase-space $(x v)$ evolution of 1-d systems is described by the one-dimensional Vlasov equation (Alard and Colombi, 2005 and references therein)

$$
\frac{\partial f}{\partial t}+v \frac{\partial f}{\partial x}+a(x) \frac{\partial f}{\partial v}=0
$$

Eq.1 
where $f(x, y)$ is the phase space distribution function and $a(x)$ is the acceleration due to selfgravitation. For 1-d systems the gravitational force is range-independent so the acceleration is given simply by $a(x)=-g\left(M_{>}-M_{<}\right)$, where $M_{>(<)}$is the total mass to the right (left) of $x$, and $g$ is the 1-d gravitational constant.

Studies of the 1-d model have a long history (Camm, 1950). These studies focus usually focus on either cosmological problems (instabilities of uniform density) (e.g. Miller et al., 2007), or isolated self-gravitating systems (e.g. Schulz, 2013). Most of the analyses of isolated systems has been performed using action-angle variables $(E, t)$ from which a general solution can be obtained, at least of the linearized Vlasov equation (e.g. Barre et al., 2011). But the calculations are rather cumbersome and opaque, and physical quantities are not easily analyzed in this approach.

We have found another way to analyze the linearized Vlasov equation, by considering perturbations of thermal equilibrium, i.e. $f=f_{0}+\delta f$. In contrast to the 3-d case, the 1-d case possesses a bound state of thermal equilibrium that maximizes entropy

$$
f_{0}(x, v)=A e^{-v^{2} / 2 \sigma^{2}} \operatorname{sech}^{2}\left(g M x / 2 \sigma^{2}\right)
$$

where $\sigma$ is the velocity dispersion and $A$ is a normalization constant. After linearizing the Vlasov equation about $f_{0}$, one can expand the perturbations in terms of Hermite (in velocity) and Legendre polynomials (in position) in the form

$$
\delta f(x, v, t)=f_{0}(x, v) \sum_{m, n} c_{m n}(t) G_{m n}(x, v)
$$

where the polynomials the $G_{m n}$ are a complete set of orthogonal normalized polynomials,

$$
G_{m n}(x, v)=\sqrt{\frac{2 n+1}{2^{m} m !}} H_{m}(v) P_{n}(\tanh (x))
$$

( $x$ and $v$ have been rescaled), and the $c_{m n}(t)$ are time-dependent expansion coefficients. The equations of motion of the coefficients are then given by the linear system

$$
\frac{d}{d t} c_{m n}=\sum_{i j} L_{m n}^{i j} c_{i j}
$$

where the couplings $L_{m n}^{i j}$, determined from Eq.1 are only between neighboring coefficients (i.e. $i, j=m \pm 1, n \pm 1)$, and are time-independent. Thus, the original phase space $(x, v)$ evolution is transformed into an evolution of coefficients on the positive $(m, n)$ quarter plane (Fig. 1). The 
initial value is solved by projecting the initial perturbation onto the orthonormal polynomials and evolving the system according to Eq.5. These equations define a very rich model whose physical properties are easy to investigate. We have been able to derive $1^{\text {st }}-$ and $2^{\text {nd }}$-order expressions for kinetic and potential energy as well as fine- and coarse-grained entropy.

Alternatively, the system can also be analyzed as an eigenvalue problem

$$
i \omega_{k} C_{k}=L C_{k}
$$

with a spectrum of real frequencies $\left\{\omega_{k}\right\}$ and complete set of eigenvectors $C_{k}$. This approach reveals a $\omega=0$ subspace of eigenvectors corresponding to the time-independent solutions of Eq.5, (and hence of Eq.1). These time-independent solutions form the basis of equilibria states that result from relaxation. In this picture, relaxation is viewed as a de-phasing of all the $\omega \neq 0$ components in the initial conditions.

Unfortunately, truncation of the series to some $\left(m_{\max }, n_{\max }\right)=(M, N)$ domain is a non-trivial problem, because reflections occur at the truncation boundaries. We have tried several approaches, including fixed boundaries, absorbing boundaries, and or small amounts of damping, but none are completely satisfactory and their impact on the time-dependent solutions is still not fully understood. Fortunately, the truncation problem can be side-stepped by considering the time-independent solutions.
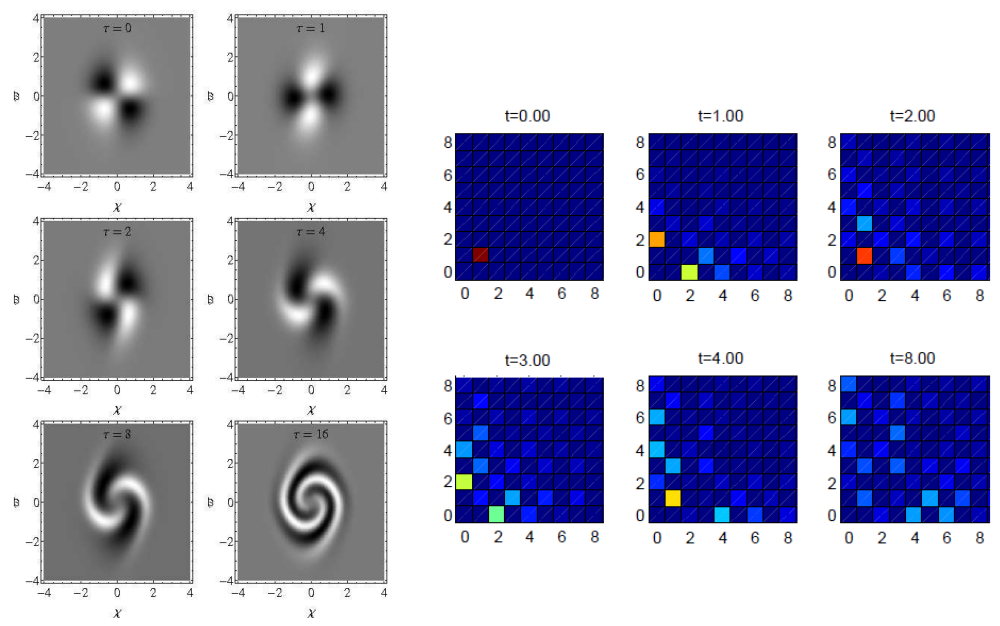

$t=3.00$
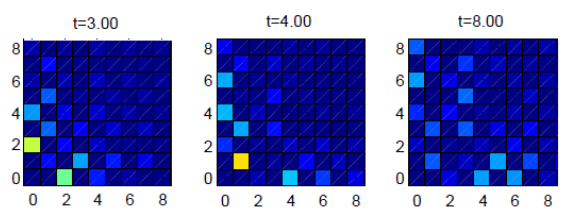

Figure 1 Comparison of evolution in phase space $(x, p)$ and coefficient space $(\mathrm{m}, \mathrm{n})$.

\section{Time-independent solutions}

The focus of this work was the subspace of time-independent solutions, which allows us to predict the final state from the initial state, but also allows us to study the redistribution of energy and entropy resulting from relaxation in a very precise way. 
The time-independent solutions can be obtained in a variety of ways. One can simply diagonalize Eq.6 numerically to obtain approximate solutions, but this approach suffers from the truncation effects at the $m=M$ and $n=N$ boundaries. Alternatively one can solve Eq. 5 with $\frac{d}{d t} c_{m n}=0$ directly, and this results in recursion relations for the time-independent solutions. Since there are an infinite number of degenerate solutions spanning the subspace, they have to be orthogonalized via a Gram-Schmidt procedure (Fig. 2). The solutions naturally truncate at some $m_{\max }$ which serves as an index. There is an additional complication in that $L$ is not Hermitian, but this has been overcome with the use of left-and right-sided eigenvectors.

One can also obtain exact solutions by expanding the velocity in a Hermite series and solving the resulting (coupled) differential equations for $\delta f$ directly. This approach is cumbersome but provides a benchmark for comparison with approximate solutions.

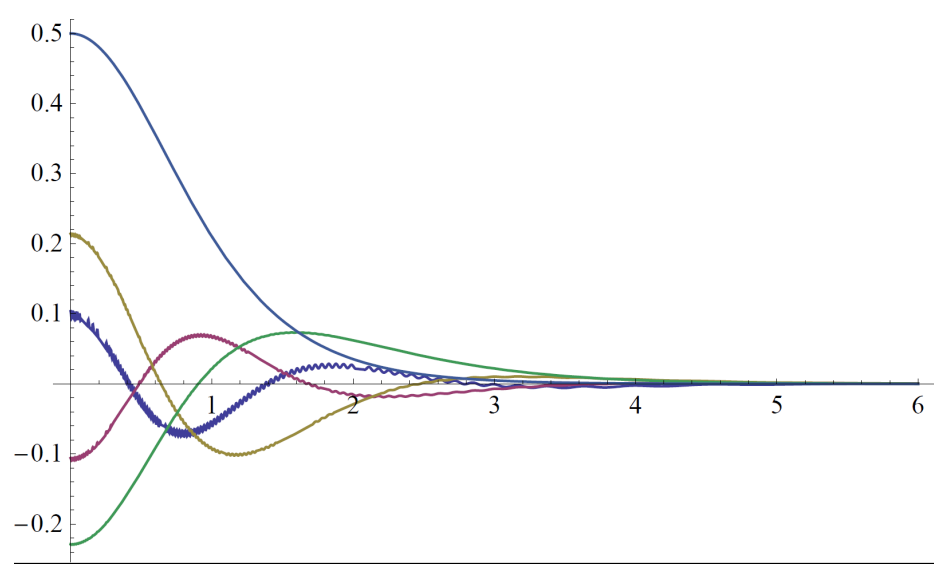

Figure 2. Density profiles of orthogonalized time-independent solutions.

Once a suitable set of orthonormal time-independent solutions has been obtained, the final states will be compared with 1-d N-body solutions for a variety of initial conditions (Fig. 3). This process should allow us to identify different classes of initial conditions according to what final states they project into. In particular, it will be interesting to see how these final states relate to the family of distribution functions obtained by maximizing coarse-grained entropy using Lynden-Bell statistics (Lynden-Bell, 1967), thus yielding a very stringent test of their applicability. 

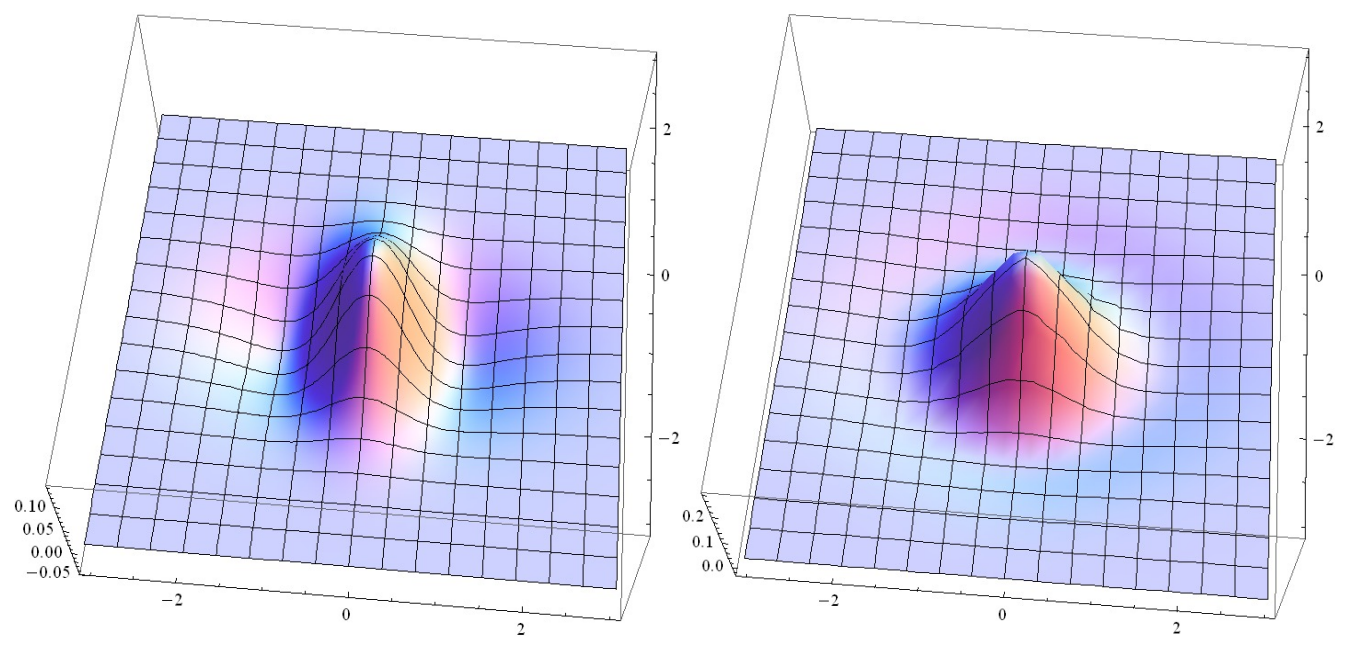

Figure 3. Example of initial conditions (left) and corresponding steady- state solution (right).

\section{Summary}

To summarize, the goals of this work were: to perform a complete analysis of the timeindependent subspace of solutions of isolated 1-d self-gravitating systems that have been slightly perturbed from the state of maximum entropy, $f_{0}$. This involved: (1) obtaining these solutions in various representations (as eigenvectors, recursive solutions, and exact solutions of coupled differential equations), (2) using these solutions to predict the equilibrium state for different initial conditions, (3) analyzing and interpreting the redistribution of energy and fine-grained entropy in the evolution of these systems from their initial conditions, (4) extending the calculations to $2^{\text {nd }}$-order to study the behavior of correlations, and (5) performing $N$-body simulations to verify our predictions and to determine their range of validity.

\section{REFERENCES recommend journal abbreviations be more than single letter words}

Alard C., Colombi S., 2005, MNRAS, 359, 123

Barnes, E. I., Ragan, R. J. 2014, MNRAS, 437, 2340 This reference is not cited in text.

Barr'e J., Olivetti A., Yamaguchi Y.Y., 2011, J. Phys. A, 44, 405502

Camm G.L., 1950, MNRAS, 110, 305

Clowe D., Bradac M., Gonzalez A.H., Markevitch M., Randall S.W., Jones C., Zaritsky D., 2006, ApJ, 648, L109

Hansen S.H., Moore B., 2006, New Ast., 11, 333

Hohl \& Felix, 1967 this reference is cited but not in references

Lithwick Y., Dalal N, 2011, ApJ, 734, 100 
Lynden-Bell, D. 1967, MNRAS, 136, 101

Miller B.N., Rouet J., Le Guirriec E., 2007, Phys. Rev. E, 76, 6705

Navarro J.F., Frenk C.S., White S.D.M., 1997, ApJ, 490, 493

Navarro J.F. et al., 2004, MNRAS, 349, 1039

Ragan, R. J., Mullin, W. J., Wiita, E. B. 2005, J. Low Temp. Phys., 138, 693 this reference not cited in text

Rubin V.C, Ford W.K., 1970, ApJ, 159, 379

Schulz A.E., Dehnen W., Jungman G., Tremaine S., 2013, MNRAS, 431, 49

Spergel D.N. et al., 2003, ApJS, 148, 175

Taylor J., Navarro J., 2001, ApJ, 563, 483

Zwicky F., 1937, ApJ, 86, 217 